\title{
Extended Release Intraruminal Device Dosage Form
}

National Cancer Institute

\section{Source}

National Cancer Institute. Extended Release Intraruminal Device Dosage Form. NCI

Thesaurus. Code C87538.

A pharmaceutical dosage form comprised of extended release agents for intraruminal administration. 